\title{
Experiment Reconstruction Reduces Fixation on Surface Details of Explanations
}

Samuel Lau

Design Lab, UC San Diego

La Jolla, CA 92093

lau@ucsd.edu
Scott Klemmer

Design Lab, UC San Diego

La Jolla, CA 92093

srk@ucsd.edu

\section{Tricia Ngoon}

Design Lab, UC San Diego

La Jolla, CA 92093

tngoon@ucsd.edu

Vineet Pandey

Design Lab, UC San Diego

La Jolla, CA 92093

vipandey@ucsd.edu

Permission to make digital or hard copies of part or all of this work for personal or classroom use is granted without fee provided that copies are not made or distributed for profit or commercial advantage and that copies bear this notice and the full citation on the first page. Copyrights for third-party components of this work must be honored For all other uses, contact the owner/author(s).

Copyright held by the owner/author(s).

C\&C '19, June 23-26, 2019, San Diego, CA, USA

ACM 978-1-4503-5917-7/19/06.

https://doi.org/10.1145/3325480.3326582

\begin{abstract}
Misunderstandings of science affect many lives. Novices commonly misunderstand explanations by overly relying on surface details instead of evaluating underlying logic. Prior work has found adding a patina of neuroscience leads readers towards positively assessing explanations. How might we help people better understand science explanations? A between-subjects experiment tested whether asking readers to reconstruct experiments leads them to focus more on underlying logic. Participants relied less on irrelevant surface details when reconstructing experiments. However, this did not impact their subsequent assessment of explanations. Our results suggest that reconstruction is a usefu strategy for understanding explanations but is not readily transferred towards evaluating explanations.
\end{abstract}

\section{Author Keywords}

fixation; science explanations; surface features

\section{CCS Concepts}

-Applied computing $\rightarrow$ Computer-assisted instruction

\section{The Seduction of Details Over Structure}

The knowledge-intensive and creative nature of modern life forces people to evaluate statements about domains they don't know much about. Misunderstandings cause problems. For example, a 1993 study found that college stu- 
Explanation: Information about stereotypical animals is stored in a certain way by CA3 brain cells, which have been shown to mediate memory. This makes the information more readily accessed and manipulated than information about rare animals.

Recall: Based on the explanation above, why was one type of animal easier to reason about than another?

Reconstruction: Suppose you are a scientist recreating this experiment and find similar results. Why might your subjects be better at reasoning about stereotypical animals than rare animals?

\section{Table 1: Comprehension}

explanation and questions for each condition. Participants also read a description of the experiment (not shown). dents momentarily performed better on spatial reasoning when listening to Mozart [6]. This "Mozart Effect" experienced widespread acceptance. Florida passed a bill requiring day care programs to play classical music to infants, Georgia budgeted $\$ 105,000$ to distribute classical music to newborns, and "Mozart Effect" products sold millions of copies [1]. The Mozart Effect paper only reported a temporary increase in spatial reasoning, yet numerous news articles claimed that Mozart makes people permanently "smarter". Compelling surface details like Mozart's name can overshadow the logic within an explanation.

Fixation on Surface Details Prevents Understanding Most people are science novices; despite the presence of scientific findings in the news, most people lack the skills to interpret or evaluate these findings. Novices focus primarily on surface details of a situation: the literal objects, concepts, or entities explicitly described [4]. As the Mozart Effect demonstrates, surface details act as fixating cues that can result in misunderstandings.

One prior study presented people with logically coherent and illogically circular science explanations. People generally perceived logical explanations as more satisfying than illogical ones. However, when irrelevant brain-related terminology was added to the explanations, novices in neuroscience rated illogical explanations more satisfying [8].

Such fixation hinders creative problem-solving by preventing broad search of a solution space [7]. When understanding a science explanation, fixation on surface details like neuroscience terminology may discourage people from examining the explanation's logic.

\section{Reducing Fixation on Surface Details}

Redoing someone else's work is a common learning strategy in creative disciplines, from painting to programming.
This work investigates whether re-explaining a scientific finding increases understanding and reduces fixation on surface details. Learning may depend on the specific type of re-explanation. Specifically, repeating an explanation word-for-word is predicted to be less effective than drawing from personal knowledge to reconstruct an explanation [3]. In the latter, people extend the underlying logic of the explanation instead of relying on its surface details. The interpretive process of constructing personal knowledge is a form of personal or mini-c creativity [5], and orients learners towards higher levels of Bloom's taxonomy [2].

\section{Can Reconstruction Improve Understanding?}

Participants were shown a science explanation from [8]. The Reconstruction group was asked to imagine themselves as scientists reconstructing the described experiment, while the Recall group was asked to base their responses on the given text (Table 1). This study compares the effect of these two tasks on understanding science explanations that contain irrelevant neuroscience terminology.

We hypothesized that Reconstruction participants would avoid fixating on the surface details in the reconstructed explanation. We also hypothesized that Reconstruction participants would also avoid fixating on surface details in subsequent explanations, even when not explicitly instructed to reconstruct them.

\section{Method}

Participants

Undergraduates were recruited from social science courses at a California research university ( $n=72,54$ female). Participants received course credit for participation and were informed that the results of their experiment would have no impact on their class performance. 
Description (excerpt): The researchers discovered that words spoken soon after a presented target word were words that sounded like the target, while words spoken later were words that had a similar meaning to the target.

Rate the quality of the following explanation:

\section{Patterns of brain activation in} these subjects lead researchers to conclude that this happens because Broca's area, a part of the brain's language system, associates two different types of words with the target word at two different times.

Table 2: Example of a circular explanation with neuroscience in the Ratings task.

\section{Design}

Participants completed an online study with two tasks: Comprehension and Ratings. There are two conditions for each task, resulting in a $2 \times 2$ design: Recall vs. Reconstruction $\times$ Without Neuroscience vs. With Neuroscience.

\section{Materials}

In the Comprehension task, participants read a description and explanation of an experiment. The explanation included a mechanism for the experimental result and an irrelevant neuroscience surface detail. In the Reconstruction condition, participants were asked to imagine themselves as scientists reconstructing the experiment and answer questions about their results. Participants in the Recall condition, on the other hand, answered questions about the text itself ( $\mathrm{Ta}$ ble 1).

In the Rating task, participants rated the quality of explanations copied from [8] (Table 2). Each explanation either proposed a logical mechanism or provided a circular restatement of a psychology finding. Each subject rated 4 logical and 4 circular explanations in a random order. In the With Neuroscience condition, irrelevant neuroscience information was added to every explanation.

\section{Measures}

Independent variables are the Comprehension task questions (Recall vs. Reconstruction), explanation content (With Neuro vs. Without Neuro), and explanation quality (logical vs. circular).

Dependent variables are the Comprehension responses and numeric ratings of explanation quality ranging from +3 (good) to -3 (bad).

One independent rater coded each Comprehension response on three binary scales: Neuro, True, and Guessed.
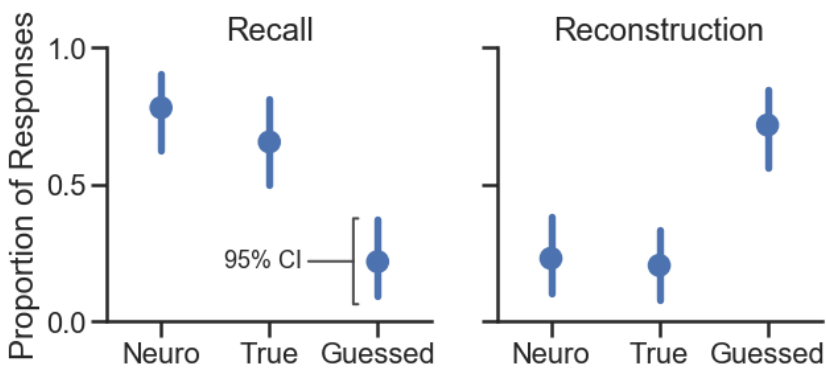

Figure 1: In the Comprehension task, Recall participants utilize irrelevant neuroscience information, while Reconstruction participants propose alternative mechanisms without relying on neuroscience information.

A response was marked as Neuro if it referenced the irrelevant neuroscience information from the original explanation. A response was marked as True if it referenced the mechanism provided by the original explanation. Finally, a response was marked as Guessed if it proposed an alternative mechanism not directly present in the original explanation.

\section{Results}

Reconstruction Participants Generate Alternative Mechanisms In the Comprehension task, Recall participants relied on the explanation's text. When asked why an experimental finding occurred, they often included the explanation's provided mechanism but also its irrelevant neuroscience information (Figure 1). Compared to Recall, Reconstruction participants were less likely to include the explanation's mechanism and neuroscience information (True: $t(61.0)=$ 4.19, $\mathrm{p}<0.01$; Neuro: $t(66.7)=5.45, \mathrm{p}<0.01)$. Instead, Reconstruction participants generated alternative mechanisms more often than Recall (Guessed: $t(68.0)=-4.79$, $\mathrm{p}<0.01$ ). This provides evidence that reconstructing an explanation encourages creative problem-solving. 


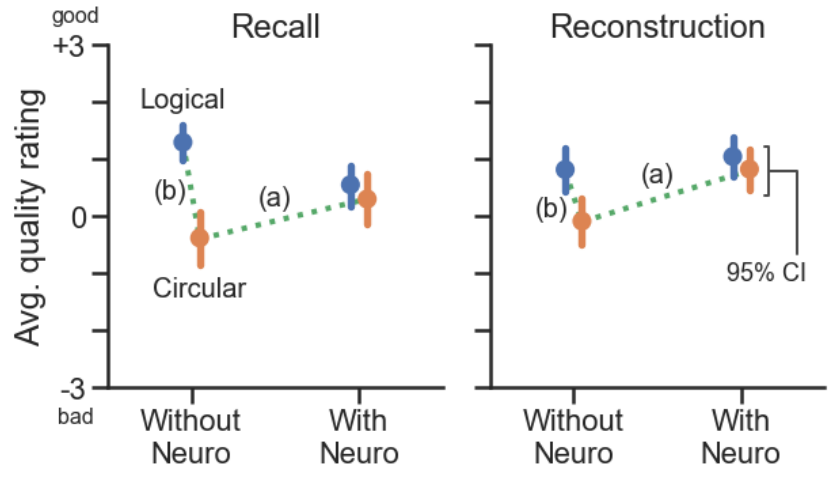

Figure 2: Contrary to our hypothesis, participants in both conditions rated circular explanations higher quality when explanations contained neuroscience information (a). Participants rated circular explanations without neuroscience lower quality than logical explanations (b).

Neuroscience Detail Increases Ratings of Circular Explanations Contrary to our hypothesis, participants in both conditions rated circular explanations with neuroscience higher quality than circular explanations without neuroscience ( $R e$ call: $t(125.6)=-2.10, \mathrm{p}<0.05 ;$ Reconstruction: $t(157.2)=$ -3.233, $\mathrm{p}<0.01$ ) (Figure 2a). In addition, there was no significant difference between Recall and Reconstruction for ratings of circular explanations with neuroscience $(t(121.8)$ $=-1.8, p>0.05)$. These patterns are consistent with prior work that did not include a task before ratings, suggesting that neither Recall nor Reconstruction mitigated the positive bias caused by neuroscience surface details.

Without Neuroscience Detail, Logical Explanations Rated Higher Quality

When explanations did not include neuroscience, participants rated logical explanations higher quality than circular explanations (Recall: $t(119.1)=5.69, p<0.01$; Reconstruction: $t(180.4)=3.22, p<0.01)$ (Figure $2 b)$. This is also consistent with prior work, suggesting that participants perceived a difference between logical and circular explanations when neuroscience was not included.

\section{Discussion}

Reconstruction Reduced Fixation on Irrelevant Surface Details Despite the inclusion of an irrelevant neuroscience surface detail in the explanation, Reconstruction participants seldom used this detail when reconstructing the explanation. In fact, Reconstruction participants proposed alternative mechanisms instead of referencing either neuroscience detail or original mechanism in their explanations. One interpretation is that these participants ignored the text entirely. If this was the case, the proposed mechanisms should be irrelevant or inconsistent. Most Reconstruction participants, however, proposed relevant mechanisms that accounted for the specific experimental results discussed in the text. Some proposed mechanisms referenced the original explanation and elaborated on it, demonstrating both knowledge of and ability to extend the text's structure. While $R e-$ call responses were often word-for-word copies of the text, Reconstruction responses proposed a variety of distinct mechanisms. For example, one Reconstruction participant suggested a mechanism based on confidence:

"it may be possible that not only is this information easier to access, but participants are more confident because of their familiarity with stereotypical birds."

Another suggested a mechanism based on prior knowledge: 
"people are more likely to have previous knowledge on stereotypical birds which makes it easier to understand the new information."

This finding suggests that reconstructing an explanation reduces fixation and encourages flexible problem-solving.

\section{The Challenge of Transfer}

Reconstruction participants used irrelevant neuroscience details as an indication of quality in the Ratings task despite avoiding the same details in the Comprehension task. Although the two tasks shared the same explanation format, participants might have perceived that the tasks were unrelated-participants might not have transferred strategies learned in one task to the other. This finding highlights the challenge of transfer even between activities that appear similar.

\section{Conclusion}

We examined whether reconstructing an explanation improved understanding of science explanations compared to a recall activity. Participants reconstructing an experiment fixated less on surface details and showed more flexibility in problem-solving. When evaluating explanations, however, participants in both conditions were still influenced by irrelevant neuroscience surface features. Although reconstruction appears to be an effective strategy for understanding explanations, participants do not readily transfer this strategy towards evaluating explanations.

\section{Acknowledgments}

We thank the UC San Diego Design Lab. This work was funded in part by NSF award \#1735234.

\section{References}

[1] Adrian Bangerter and Chip Heath. 2004. The Mozart Effect: Tracking the Evolution of a Scientific Legend. British Journal of Social Psychology 43, 4 (2004), 605-623. 00254.

[2] Benjamin S. Bloom. 1956. Taxonomy of Educational Objectives. Vol. 1: Cognitive Domain. New York: McKay (1956), 20-24. 31958.

[3] Michelene TH Chi. 2009.

Active-Constructive-Interactive: A Conceptual Framework for Differentiating Learning Activities. Topics in cognitive science 1, 1 (2009), 73-105. 00840.

[4] Michelene TH Chi, Robert Glaser, and Ernest Rees. 1981. Expertise in Problem Solving. Technical Report. PITTSBURGH UNIV PA LEARNING RESEARCH AND DEVELOPMENT CENTER. 02932.

[5] James C. Kaufman. 2009. Beyond Big and Little: The Four C Model of Creativity. 01276.

[6] Frances H. Rauscher, Gordon L. Shaw, and Catherine N. Ky. 1993. Music and Spatial Task Performance. Nature 365, 6447 (1993), 611. 01567.

[7] Steven M. Smith and Steven E. Blankenship. 1991. Incubation and the Persistence of Fixation in Problem Solving. The American journal of psychology (1991), 61-87. 00450.

[8] Deena Skolnick Weisberg, Frank C. Keil, Joshua Goodstein, Elizabeth Rawson, and Jeremy R. Gray. 2008. The Seductive Allure of Neuroscience

Explanations. Journal of cognitive neuroscience 20, 3 (2008), 470-477. 00969. 